RIEF Revue italienne d'études françaises

Littérature, langue, culture

5 | 2015

Varia

\title{
Un livre blanc de Philippe Vasset ou le silence des cartes
}

Ida Porfido

\section{(2) OpenEdition}

Journals

Édition électronique

URL : http://journals.openedition.org/rief/1038

DOI : 10.4000/rief.1038

ISSN : 2240-7456

Éditeur

Seminario di filologia francese

Référence électronique

Ida Porfido, «Un livre blanc de Philippe Vasset ou le silence des cartes », Revue italienne d'études françaises [En ligne], 5 | 2015, mis en ligne le 15 décembre 2015, consulté le 19 avril 2019. URL : http:// journals.openedition.org/rief/1038; DOI : 10.4000/rief.1038

Ce document a été généré automatiquement le 19 avril 2019

\section{(c) (i) (9)}

Les contenus de la RIEF sont mis à disposition selon les termes de la Licence Creative Commons Attribution - Pas d'Utilisation Commerciale - Pas de Modification 4.0 International. 


\title{
Un livre blanc de Philippe Vasset ou le silence des cartes
}

\author{
Ida Porfido
}

1 Dès la fin du XVIII ${ }^{e}$ siècle, quand elle a été promue "capitale de [ce] siècle ", la ville de Paris a été parcourue, balisée, déchiffrée tel un texte par de nombreux écrivains français avides d'expérimentations en tout genre. Ce phénomène, encore bien présent au siècle dernier, au point de constituer un véritable topos littéraire, inclut habituellement les flâneries baudelairiennes, les visites dadaïstes, les déambulations surréalistes, l'«infraordinaire» perecquien, les «dérives» situationnistes et les contraintes oulipiennes. Toutefois, la littérature plus récente décline elle aussi, avec force nouveautés et variations, le thème complexe de la ville et de ses alentours ${ }^{1}$. Centré sur Un livre blanc de Philippe Vasset (Fayard, 2007), notre travail tentera de montrer l'intérêt et la fascination que ce texte produit aussi bien d'un point de vue purement littéraire que dans une perspective plus vaste, à la fois géographique, sociologique, philosophique, voire politique.

2 Nous partirons d'une réflexion de Frédéric Martin-Achard sur le concept de banlieue, entendue comme image renversée de la ville ${ }^{2}$, lieu interstitiel exerçant une très forte attraction sur de nombreux artistes (des photographes surtout, souvent américains) dès les années 1970. Pour ce qui est de la littérature française, en revanche, il faut attendre les décennies suivantes pour assister à un renversement de perspective susceptible de mettre les marges au centre d'une exigence esthétique pointue. Dès les années 1990, en fait, on voit apparaitre un véritable sous-genre narratif que le chercheur appelle le « récit périurbain $»^{3}$. Tous les auteurs auquel il se réfère (François Bon, Jean Rolin et Philippe Vasset) partagent «une approche non-fictionnelle de la banlieue, vécue et rapportée souvent de façon empirique et subjective $»^{4}$. Pour eux, appréhender le paysage signifie relater une expérience personnelle $\mathrm{du}$ réel, car le récit qui en résulte donne inévitablement un sens à des lieux qui sont la plupart du temps « abandonnés à l'aridité des données ou relevés topographiques $»^{5}$. Vasset, comme nous le verrons, a cherché lui aussi à renouveler la pratique de ce sous-genre narratif par l'invention d'un type 
d'enquête urbaine inédit qui, tout en mettant la littérature en relation à d'autres savoirs et discours (il suffit de penser que le titre de l'œuvre est accompagné d'un sous-titre, « récit avec cartes »), se montre capable d'utiliser les artifices de la parole littéraire pour aller jusqu'aux confins du "dicible». En cela, son entreprise semble faire écho à la démarche novatrice suivie par Jacques Lévy depuis quelques années. Son postulat est bien simple : le monde dans lequel nous vivons est mal connu, mal appréhendé par les sociétés, notamment dans sa présentation ou sa lecture cartographique, les distances kilométriques ne correspondent plus à notre réalité actuelle, le territoire est dépassé sans cesse par l'espace dont la base n'est plus le métrique mais les liens entre les hommes ${ }^{6}$. Mais procédons dans l'ordre et commençons par la genèse du texte objet de cette étude.

\section{Brève chronique en guise de prémisse}

3 Pour Vasset, la carte géographique «entretient des relations fort lointaines avec la réalité $»^{7}$ : elle révèle un paysage idéal, vu d'en haut, donnant ainsi une représentation totalisante et privée de point de vue (si ce n'est celui de Dieu) ${ }^{8}$. " La carte dit qu'il n'y a rien derrière, mais cela est difficile à croire $»^{9}$. Il y a quelques années, Vasset a donc commencé à examiner à la loupe la carte $n^{\circ} 2314$ OT de l'IGN qui comprend Paris et sa banlieue. Laissant derrière lui l'ordre haussmannien du centre-ville, représenté en détail par des dizaines de signes et de symboles conventionnels, il a suivi les voies ferrées et les lignes de métro pour se porter jusqu'aux limites de la ville où, après le périphérique, commence l'hinterland de la capitale française. C'est justement dans ces zones, « avantpostes de la modernité $»^{10}$, comme il les a définies, qu'il a découvert de véritables terrae incognitae, presque des no man's lands, car " plutôt que surcharger le dessin et rompre les proportions avec des symboles compliqués », explique-t-il dans l'incipit d'Un livre blanc, " les cartographes laissent parfois certaines zones vierges " ${ }^{11}$, mystérieusement blanches, privées de toute inscription. Que se cache-t-il alors derrières ces "occultations suspectes » : zones militaires, sols soumis à la spéculation immobilière, lieux de rendezvous obscurs, quoi d'autre encore ? Comment remplir l'absence évidente d'information et de connaissance qui semble caractériser ces zones abandonnées, hybrides, ces étendues de terrains emblématiquement définis comme vagues en français ${ }^{12}$ ?

Convaincu que le visible doit être façonné et qu'il n'est pas donné une fois pour toutes, l'écrivain a décidé de les visiter personnellement, de les sillonner et d'en faire l'expérience avec son corps : à pied, sens en alerte. Tel un nouvel explorateur, il s'est promené avec curiosité dans ces lieux réfractaires à toute découverte, en se dotant de moyens technologiques sophistiqués (GPS, appareil photo, caméra vidéo, téléphone portable, différents instruments d'enregistrement, etc.), mais aussi des supports d'écriture les plus traditionnels (carnet et stylo) pour documenter avec précision l'existant, jusque dans ses changements les plus infimes. Pendant plus d'un an, de weekend en week-end, il a ainsi parcouru systématiquement une cinquantaine de fragments de cette banlieue, revenant plusieurs fois sur les mêmes zones après quelque temps ${ }^{13}$, et il a tenté d'en faire le rapport «à chaud» comme à «froid». Son projet, aventureux dans tous les sens du terme, parfois vécu comme un véritable devoir auquel il ne peut se soustraire - « je prenais ma mission très au sérieux $»^{14}-$, constitue le point de départ du Livre blanc et le matériau brut dont il est fait. En effet, sa structure formelle et narrative est très hétérogène et décousue : en plus des cartes, partie intégrante du texte, le récit comprend des extraits de notes prises durant ces expéditions, des fragments isolés qui 
semblent réélaborer de façon créative les « découvertes » faites, des bribes de réflexions sur le paysage environnant ou sur l'avancée du discours en général, souvenirs, visions, considérations impromptues, va-et-vient mentaux...

$5 \quad$ Les dix-sept zones sélectionnées apparaissent comme de véritables trous dans les mailles du réel familier, de petits amas d'inconnu dont l'auteur sent le besoin irrésistible de rendre compte par diverses stratégies. Et son récit débute justement là où la carte se heurte au non représentable, reste obtuse, muette. Le texte se déploie ainsi dans le vide laissé par la carte, profitant de son incapacité à accueillir et à fixer les traits distinctifs d'une réalité « impraticable » aux yeux du technicien, pour nous la restituer par un récit que nous pourrions définir comme " animé $»^{15}$. Dans Un livre blanc, la description pseudoobjective alterne avec des passages (généralement en italique) qui donnent une image fragmentée du réel, souvent plongée dans une atmosphère suspendue, raréfiée, presque onirique. La plupart du temps, il s'agit de notes prises sur place à la hâte, sur le vif, transformées ensuite en narration. Elles enregistrent des rencontres fugaces avec des personnes, des animaux, des plantes, des situations, des scénarios qui, pour une raison ou une autre, ont frappé l'écrivain: occasions exceptionnelles, moindres coïncidences, minuscules épiphanies. Plus généralement, l'écriture de Vasset tend à s'échapper du caractère purement factuel des choses et à fuir l'agencement textuel. Peu importe le « postulat » dominant (plus enclin à la fiction ou collant davantage à la réalité) : sa prose semble dépasser le texte pour surgir $d u$ réel et dans le réel, se faire graphe, geste. Et cela représente sans doute l'aspect le plus intéressant de son travail.

Ainsi dans Un livre blanc, cartographie et écriture ne s'entrecroisent pas seulement pour composer un « documentaire narratif » fort original, un récit littéralement accompagné de cartes, comme l'entend le sous-titre indiqué par l'auteur. La rencontre entre ces deux pratiques donne vie à une expérience euristique et esthétique singulière. «Je ne savais pas où m'aurait porté exactement le texte ", confesse Vasset au début du récit, et le livre ressemble, en effet, avec ses trajets et ses haltes, à une performance artistique centrée sur la recherche d'une forme, de la Forme, plus qu'à un compte rendu ou à un journal de voyage traditionnels ${ }^{16}$.

\section{La fabrique du texte}

7 La raison principale des incursions de Vasset, peut-être un peu trop ingénue bien que franchement autobiographique, est la recherche du merveilleux urbain. « Au cours de cette quête, j'espérais, comme les héros de mes livres d'enfant, mettre au jour le double fond qui manquait à mon monde $»^{17}$, écrit-il dans les premières pages du livre.

8 Mais derrière les barrières défoncées, les grillages troués, les murs écroulés qui délimitent son champ d'enquête, et une fois entré - souvent par effraction -, dans ces zones frontières de la ceinture urbaine, l'écrivain ne trouve que les débris abandonnés d'une «modernité liquide» en expansion ${ }^{18}$. C'est que les villes modernes repoussent loin du centre, vers l'extérieur, hors d'elles, les usines abandonnées, les montagnes de déchets, les ruines insignifiantes, les trafics illégaux: tout ce qui de notre monde postindustriel est écarté, submergé, des éclats de réalité intrinsèquement changeants et sémantiquement opaques que la main négligente de l'homo oeconomicus mélange sans cesse $^{19}$. 
Cela fait penser au célèbre «non-lieu » analysé par Marc Augé, un outil herméneutique dont la critique littéraire a largement profité ces dernières années ${ }^{20}$. La banlieue y apparait comme un lieu de transit qui exclut l'être humain et empêche la construction d'un milieu social ; un lieu où les trajets individuels - la promenade par exemple -, ou bien les échanges ou les rassemblements, seraient désormais impossibles.

Pourtant, comme nous le savons, dans ces terres désolées subsistent diverses formes de vie, non seulement végétale ou animale (ce sauvage dont Vasset parle à plusieurs reprises), mais des bidonvilles entiers aux portes de Paris ainsi que des campements isolés, de simples baraques, et des lieux de rendez-vous clandestins. Il s'agit souvent de constructions de fortune, faites de bric et de broc par une humanité errante à la recherche de nouveaux habitats pour survivre entre les aspérités et les plis de notre société de consommation :

Par endroits, Paris n'était plus que caravanes et immeubles désaffectés entre lesquels serpentaient, silencieuses et résignées, des files de silhouettes immobiles attendant pendant des heures devant les préfectures, les soupes populaires et les pharmacies. ${ }^{21}$

Pas de clochards, plutôt des migrants, vraisemblablement des Roms venus de l'Europe de l'Est. ${ }^{22}$

11 L'extrême pauvreté et précarité auxquelles se heurte l'auteur évoquent en lui le souvenir de certaines photographies de bidonvilles de Nanterre pendant la guerre d'Algérie ${ }^{23}$, et lui permettent de restituer aux zones blanches une « verticalité » et une « densité » oubliées jusque-là. Car, en adoptant un procédé quasi phénoménologique destiné à constater le pur «être là » des choses, Vasset semble avoir opté, du moins au début, pour une « ignorance délibérée » : «[...] afin d'éviter d'apprivoiser paysages et constructions à coup de dates et d'anecdotes, je n'étudiais pas l'histoire des sites [...] $»^{24}$. Malgré cela, la dimension historique des sites visités parvient à pénétrer par les mailles de son récit et à remplir le soi-disant vide ambiant. Non seulement le paysage est nécessairement en relation avec l'expérience temporelle mais, comme le souligne Martin-Achard ${ }^{25}$, la question temporelle même est au centre de tout récit périurbain. De plus, souvenonsnous, le projet sous-jacent à l'écriture de ce livre possède des limites temporelles bien précises, destinées à conjurer durée et déplacement pour que le sujet spectateurnarrateur puisse juger de l'action et des effets du temps sur le paysage périurbain. Dans cette optique, les sites visités par Vasset s'avèrent fort mobiles, changeants, grouillants, éléments qui rappellent tous à l'esprit le concept clé, quelque peu suremployé dernièrement, de Zygmunt Bauman.

Les sites que je visitais étaient instables, en proie, comme un front de nuages, à une agitation perpétuelle: tout restait fuyant, à peine entrevu et, bien qu'immobile, j'étais chaque fois saisi par le satori du transit qui dérobe le monde. ${ }^{26}$

D'une exploration à l'autre, certains sites changent radicalement alors que dans d'autres Ville et Nature semblent se livrer à d'invisibles combats pour affirmer leur suprématie : flore et faune endémiques (orties, chardons, roseaux, etc./lapins, canards, renards, etc.) sont largement représentées, bien qu'immanquablement engagées dans une " résistance » exténuante ${ }^{27}$. Les zones blanches apparaissent ainsi comme des failles, aussi bien d'un point de vue spatial que temporel, à savoir "des terrains non identifiés topographiquement, dont la seule caractéristique est d'être situés entre deux espaces singularisés par la carte, et des zones laissées à la pure action d'un temps indéfini entre deux moments de l'aménagement urbain $»^{28}$. Voilà pourquoi, si surprenant que cela puisse paraître, c'est hors des villes que les mutations du paysage et de la société 
contemporaine sont le plus perceptibles. C'est aux marges que paraissent coexister des constructions en cours (zones résidentielles, centres commerciaux, etc.). et des vestiges du temps passé (friches ou sites industriels, etc).

Par ailleurs, ce type de paysage "mutant » se présente comme un champ de bataille qui renferme les traces évidentes d'une violence urbaine s'exerçant subrepticement sur les choses et les gens. À quelques centaines de mètres des mirobolants bureaux en verre des grandes entreprises et des beaux hôtels particuliers de la Ville Lumière, voilà qu'apparaît crûment l'envers du décor. Alors, plus que représenter le dévoilement du côté caché de la ville, les paysages décrits dans ce livre essaient de suggérer une reconfiguration possible des relations entre espace urbain et population.

Ainsi, la misère indigne que découvre Vasset, décidé à documenter l'indicible et à lui donner un nom et une représentation, le contraint à dépasser la surprise stérile ou la pure attraction des débuts et à réorienter son projet vers une forme plus participative et informée. Il pense alors interroger les « résidents » (Arthur, électricien polonais, Ruslan, plombier bulgare, etc.), consulter les spécialistes des disciplines affines (géographes, urbanistes, sociologues) pour mieux se rapprocher de la réalité. À partir d'un certain moment, les espaces vides des cartes - rappelons qu'en français blanc signifie aussi bien «blanc » que " vide » - deviennent donc pour lui de véritables " portes », des brèches, des entailles à travers lesquelles passer de la contemplation au savoir et à l'action.

Il s'agit de sa première tentative de faire apparaître une " autre réalité », de donner corps à un nouveau point de vue sur la ville invisible. Pourtant, le « documentaire engagé » fait misérablement faillite: "Mais lorsque j'ai voulu synthétiser toutes les informations rassemblées, les phrases ont refusé de s'agencer en argumentaire: mes textes n'expliquaient rien, ne racontaient aucune histoire ${ }^{29}$.

Désenchantement et découragement l'obligent donc à imaginer une approche différente. Abandonnant les habits de l'explorateur et du chercheur, Vasset inaugure une nouvelle phase de son enquête où perce aussi sa détermination à habiter les espaces visités, bien que de «façon provisoire»: «je voulais essayer de vivre quelque temps dans un lieu totalement inhospitalier, d'habiter l'inhabitable $»^{30}$. Pendant un certain temps, il caresse l'idée de s'installer dans un lieu vierge, le meublant avec des objets choisis pour imiter une maison normale (rideaux, nappes, bibelots, moquette, papier peint, etc.).

a nouveau, la sensation d'être un intrus, de faire litteralement irruption dans des lieux dont l'accès est contrôlé, sinon interdit, lui suggère que ces zones qui l'attirent par leur opacité appartiennent à d'autres et qu'il restera toujours un étranger au double sens du terme français. Son désir de voir le met alors inévitablement dans une situation de porte-à-faux et de voyeur ${ }^{31}$.

18 Si l'on ne peut ni le connaître ni l'occuper, comment fait-on pour parler de cet inconnu paradoxal $^{32}$ ?

19 S'offrent immédiatement à lui deux possibilités : la production de fictions capables de donner un nouvel enchantement au monde et la description minutieuse de la réalité, accompagnée du fantasme correspondant de l'exhaustivité. Dans les deux cas toutefois roman et texte - on perdrait le rapport entre les cartes et le réel qui est le postulat d'Un livre blanc dès sa conception. Et Vasset le sait.

Quant à la fiction au sens large, Vasset n'a jamais nié sa méfiance face au roman - « une forme molle comme la guimauve des sitcoms $»^{33}$. Il n'y a jamais chez lui l'intention d'écrire une œuvre purement narrative qui aurait la ville pour décor, mais celle de faire 
devenir «la ville un personnage en tant que tel, et surtout une machine narrative $»^{34}$. Pour cela, la première voie paraît impraticable dès le début.

Or, presque aussitôt, l'auteur abandonne aussi la seconde: rédiger un inventaire exhaustif ${ }^{35}$. La liste, certes, crée un effet de simultanéité et casse toute composition établie. Elle donne vie à un conglomérat d'éléments hétérogènes, mobiles et divers, tout comme le réel qui intéresse l'écrivain. Mais comment Vasset peut-il fixer cela avec les mots quand la carte échoue avec les signes? Il faudrait inventer des centaines de nouveaux symboles pour dessiner des usines abandonnées, des hangars effondrés, des squats, des lieux de rendez-vous pour les motards et bien d'autres choses encore ${ }^{36}$. Généralement, en effet, symboles et légendes désignent des éléments durables, voire permanents du paysage. Les signes sont à court de moyens devant le précaire, le fugace et bien sûr les traces les plus diverses des activités humaines. Par conséquent, en accumulant simplement des noms, l'écriture finit par se heurter à ses limites - sa finitude ,- là où elle ne fait que tracer un seuil invisible entre le dicible et l'indicible.

Cependant, en dépit des nombreuses difficultés qu'il rencontre, Vasset continue à éprouver le besoin de rapprocher le texte des zones blanches. Pendant quelque temps, par exemple, il cède à la tentation d'abandonner les notes prises sur place, l'enregistrement immédiat du réel, sur le lieu même où est advenue l'écriture, comme s'il voulait couvrir de signes ces espaces signalés par la carte comme autant de pages blanches, « porter le texte là où il n'a aucune place, où il est, au mieux, incongru, déplacé, et observer ce qui se passe ${ }^{37}$ : une écriture en même temps de ces explorations et dans ces zones vides. D'une certaine façon, comme le dit bien Martin-Achard, «derrière Un livre blanc se dessine l'utopie d'une forme de littérature in situ, de "land writing" comme on peut parler de land art $\aleph^{38}$. Vasset réalise en quelque sorte un texte-performance: l'œuvre qu'il écrit est elle-même oscillation, « équilibre instable au seuil de l'expression » 39. Et cette nouvelle forme d'écriture, qui s'affranchirait du livre pour envahir l'espace, serait rendue possible par une nouvelle technologie: «l'informatique diffuse » ou pervasive computing. Il suffirait d'associer un lieu à un texte, explique Vasset ${ }^{40}$, capable d'être diffusé sur les téléphones portables des personnes venant à passer par là.

Quel que soit l'angle de lecture envisagé, même temporel, le récit périurbain de Vasset privilégie de toute évidence une écriture "de l'espace», pouvant accueillir les lieux fragmentaires auxquels elle s'intéresse et le désordre urbain qui y règne ${ }^{41}$. Sa recherche d'un procédé dont le but est d'élargir les moyens limités que possède la langue pour dire l'espace se fait ainsi explicite ${ }^{42}$. Nous pourrions ajouter que l'on assiste à une véritable tentative de fusion entre la carte et l'écriture: «les signes cartographiques sont remplacés par leurs équivalents linguistiques et les syntagmes nominaux sont disposés sur la page comme sur le plan $»^{43}$ (on fait ici allusion aux séquences horizontales ou verticales de mots qui caractérisent la partie finale du livre). Parfois il lui arrive même de confondre les deux plans de la réalité - cette carte et ce territoire qui ne coïncident jamais -, par exemple quand il dessine par terre, à l'aide d'une bombe de peinture, les frontières des zones blanches telles qu'elles sont indiquées sur la carte : un geste aussi parlant que vain, qui aspire à opérer le renversement du monde sur le papier mais dont l'impossibilité ne révèle, en creux, que la force du geste littéraire. Même s'il retrouve ainsi une proximité momentanée avec le réel, avec ses menus détails, Vasset a l'intuition que la monotonie et le côté maniaque de ces tentatives de saturation et d' «épuisement » d'un lieu le poussent vers d'autres directions. 

choix se révèle le plus avantageux. La « transparence $»^{44} \mathrm{du}$ sujet actif permet, en effet, la production d'une cartographie différente du réel, la rupture avec des perspectives et des chemins trop souvent battus. Contrairement à ce que l'on croit ou que l'on veut nous faire croire - tout est connu, il n'y a rien d'autre, rien «de plus", ce qui revient à imposer l'idée d'un réel univoque, définitif, positif - la nouvelle méthodologie de Vasset démontre qu'il est possible d'emprunter d'autres chemins pour donner une certaine réalité (ou une réalité certaine) aux espaces «nomades » et pour en préserver la nature complexe. Pour cela, "contrairement aux rêveries et aux flâneries d'antan, il choisit une démarche volontariste où le narrateur, qui confesse s'être taillé "son petit personnage de touriste périurbain" [p. 99], s'avère en réalité un novateur à part entière, déterminé à déjouer la planification des urbanistes par l'introduction d'une pratique libre de l'espace, un point de vue "non autorisé" là où tout est dirigé et obligatoire ${ }^{45}$. Son projet a pour but de redonner un sens aux lieux abandonnés, d'y créer de nouveaux itinéraires et donc une autre visibilité et une autre présence humaines. «En restituant à la banlieue les mots dont elle est privée, Vasset cherche aussi à la rendre habitable, à rétablir un lien entre les hommes et le territoire dans lequel ils vivent $»^{46}$. Son expérience esthétique et littéraire semble alors se développer en fonction d'un impératif éthique et politique latent qui envisage, quoique de façon indirecte, la possibilité de refonder une collectivité, de reconstruire une polis ${ }^{47}$. La dernière page, surprenante jusqu'au vertige, offre une réflexion profonde sur la manière dont il faudrait habiter notre monde afin de lui donner forme et mesure humaines.

Regardez bien, vous êtes passé ici des centaines de fois : est-ce que vous savez où vous êtes et ce qui s'y passe? Privés de leur nom et de leur fonction, les bâtiments s'avachissent comme des emballages crevés [...] il n'y a que des objets incertains et des événements indécidables. Où est votre place ? Comment habiter ici? Malgré la couverture satellite permanente et le maillage des caméras de surveillance, nous ne connaissons rien du monde. ${ }^{48}$

Ce qui frappe le plus dans Un livre blanc, c'est justement l'incertitude constante sur les modalités poursuivies par la connaissance, une indécision qui semble contredire l'extrême précision dans la localisation des zones dont parle l'auteur. Les nombreuses tentatives avortées, apparemment toutes dysphoriques et décevantes, qui parsèment le récit, ne doivent pas étonner. En réalité, le temps long de l'errance et de l'attente suite à l'abandon des projets initiaux est au service de l'expérience textuelle en soi. La redéfinition répétée (qui n'est jamais une remise en cause des fondements), ce perpétuel « ajuster le tir » est une façon de choisir pour ne pas abdiquer face à la fausseté et à la banalité du discours consensuel sur les périphéries urbaines, pour résister à l'idée d'une réalité « pleine » et sans « restes ». Celle de Vasset est, à tous les égards, une expérience alternative, vouée à construire un espace différent de recherche et de création. Et il convient de souligner la cohérence du projet d'ensemble, car ce que l'auteur présente comme des tentatives sans suite sont en vérité des moments différents d'un seul et même parcours, qui demeure et résiste en dépit des déviations ébauchées vers des itinéraires alternatifs ou virtuels.

Par ailleurs, en commentant sans cesse son texte, Vasset illustre une particularité du récit périurbain qui trahit aussi sa modernité : l'abondance de métadiscours sur le dispositif narratif lui-même. Le texte se met en scène, exhibe son devenir, comme s'il s'agissait d'une machinerie aux engrenages bien en vue ${ }^{49}$. Le texte de Vasset fait le choix d'épouser le mouvement dans toutes ses manifestations, y compris les aventures à peine ébauchées, 
le vide même des rencontres manquées (lors d'une de ses incursions, le narrateur assiste de loin à une rixe, voit quelqu'un s'enfuir ou fuit lui-même devant le danger représenté par les chiens errants ou les agents de sécurité), les projets avortés. Sans ordre ni hiérarchie, le livre trace sa route au milieu des possibilités multiples qui s'offrent chaque fois, tout comme un badaud qui marcherait lentement dans ces zones sujettes à l'ensauvagement et aux « glissements » permanents.

En définitive, Vasset choisit d'enquêter sur cet espace propre à la littérature, là où la fiction rencontre le réel, en tentant de réduire coûte que coûte l'écart croissant entre les fictions dont nous sommes nourris «jusqu'à la nausée » et la réalité nue reléguée à la périphérie de notre champ visuel.

Explorant mes terrains vagues, zones vouées à la pure potentialité, lieux de l'inconfort extrême où rien ni personne n'a de place assignée, j'avais le secret espoir que les notes désordonnées et contradictoires finissent par aboutir à un texte qui ressemble à cette terre mille fois retournée et mêlée de débris, à ces toiles d'araignée qui s'accrochaient aux oreilles et aux cheveux et à ces fruits poussant sans arrosage ni jardinier..$^{50}$

L'objectif final n'est pas tant l'écriture d'un réquisitoire social - « je n'ai pas une attitude moraliste sur les zones blanches ou leur dégradation ", dit explicitement l'auteur ${ }^{51}$ qu'une tentative de changer, de déplacer nos habitudes de lecture, des livres et du réel. Dans le cadre de cette recherche, le texte n'est plus qu'une configuration temporaire de lui-même, incapable d'épuiser définitivement le lieu. En ce sens, il est aussi ou surtout une interrogation profonde et nécessaire sur le récit, sur sa capacité d'appréhender le monde et de l'exprimer à l'intérieur du mouvement qui le porte vers le monde, sa matière et son projet.

\section{Spécificité de la littérature}

Malgré ses faiblesses, ses oublis, ses imperfections et ses "vides ", l'œuvre se crée aux marges d'elle-même et de ses intentions. Elle est une zone blanche souvent traversée par le langage où se succèdent moments descriptifs dépouillés et brefs comptes rendus d'événements, ou bien des réflexions personnelles et profondes sur la relation entre corps, espace, temps et mémoire des lieux. S'adressant aux franges inexplorées du réel, l'écriture de Vasset dialogue avec le monde et revendique une forme autonome de connaissance. La littérature, en effet, et peut-être elle seulement, paraît apte à s'emparer des mots « les plus graves et les plus fragiles » et à procéder à une « resystématisation de la langue $»^{52}$, car sa force consiste justement dans le fait qu'elle réussit à dire le monde et à restituer la façon dont le perçoit l'individu. La précarité des zones explorées par Vasset et des vies qui les habitent échappent aux analyses statistiques, aux sciences «dures». Seule l'approche subjective et sensible, faite de curiosité et d'imagination, peut dévoiler la part de ce "double fond des villes ultra modernes $»^{53}$. Vasset essaie alors de percevoir le moindre son (grondement des moteurs, chants des oiseaux), la moindre image (du ciment craquelé, des murs troués) et le moindre graphème (tags, dessins) pour tenter d'en fournir le signe littéraire. Il ne s'agit pas de recouvrir le blanc, de l'annuler par le noir de l'écriture, mais bien au contraire de l'expérimenter, de le délimiter, de le dessiner, de le dire.

Cependant, l'écrivain va encore plus loin et propose une nouvelle idée, fort concrète et efficace, de texte littéraire, capable d'avoir une suite, d'être reproduit-prolongé par 
n'importe qui et de s'inscrire alors dans le territoire collectif mobile qu'est Internet. Convaincu que la littérature peut être en mesure d'inventer des pratiques plutôt que de se limiter à reproduire des objets finis, Vasset crée avec deux artistes, Xavier Courteix et Xavier Bismuth, l'Atelier de Géographie Parallèle : un vaste projet qui réunit toutes les tentatives de représentation des points aveugles de la carte $n^{\circ} 2314$ OT de l'IGN. Ces représentations, consultables sur le site http://www.unsiteblanc.com/, rassemblent photos, récits, sons, vidéos et esquisses réalisés chemin faisant.

Et pour ceux qui se plaisent à naviguer dans ces eaux métropolitaines, il existe aussi en Italie un groupe qui partage depuis longtemps ce même intérêt. Il s'agit du «Laboratorio di arte urbana Stalker/Osservatorio nomade " qui parcourt en priorité la banlieue de Rome et chasse les histoires aux/dans les marges de la ville ${ }^{54}$.

\section{NOTES}

1. Cf. Papier-villes, coordonné et préfacé par M.T. Jacquet, Bari, B.A. Graphis, 2008, ainsi que le livre de Filippo Zanghi, Zone indécise. Périphéries urbaines et voyage de proximité dans la littérature contemporaine, Lille, Presses Universitaires du Septentrion, 2014.

2. Cf. F. Martin-Achard, " "Des promenades dans cette épaisseur des choses reconstruites". Introduction au récit périurbain (Bon, Rolin, Vasset) », dans Comparaison, 1, 2008, p. 5-25.

3. Ibid., p. 5. Le concept de périurbanisation tel qu'il a été défini par Michel Lussault sied parfaitement avec l'analyse des réalités spatiales dont il sera question ici. En effet, le géographe met très souvent l'accent, surtout dans ses études les plus récentes, sur le paradoxe d'une urbanisation contemporaine marquée certes par l'étalement matériel mais aussi par l'importance grandissante de ces vides qui font la ville. De ce fait, les espaces périurbains se présentent à lui comme des espaces de diffusion où la dissipation est tout aussi signifiante que la croissance. Voir M. Lussault, L'homme spatial, Paris Seuil, 2007 et L'avènement du monde. Essai sur l'habitation humaine sur la terre, Paris, Seuil, 2013.

4. Ibidem.

5. Ibid., p. 6.

6. Cf. le corpus des écrits de Jacques Lévy, dont notamment Le tournant géographique, Paris, Belin, 2000.

7. Ph. Vasset, Un livre blanc, Paris, Fayard, 2007, p. 9.

8. Les cartes trompent notre conception de la réalité parce qu'elles s'éloignent de la représentation vraie, comme l'explique M. Onfray : "Le monde n'est pas ce qui paraît car le centre de gravité des projections nous trompe avec des fictions. Une carte énonce l'idée qu'on a du monde, pas sa réalité » (Théorie du voyage. Poétique de la géographie, Paris, Librairie Générale Française, 2011, p. 29). Sur le même sujet, voir aussi Henri Garric, Portraits de villes. Marches et cartes : la représentation urbaine dans les discours contemporains, Paris, Honoré Champion, 2007, où l'auteur souligne la tension qui existe entre la linéarité de la langue et la simultanéité de la carte dans toute tentative de représentation/invention de la ville.

9. Ph. Vasset, op. cit., p. 13.

10. R. Pasquier, «La ville et ses fictions. Rencontre avec Bruce Bégout et Philippe Vasset ", dans Papier-villes, cit., p. 81-82. Notre auteur est persuadé que «la frontière du monde connu passe désormais aux portes de la ville. Les mégapoles s'indifférencient sur leurs marges, et les zones 
blanches sont [...] les points par où Paris, Lagos et Rio communiquent comme les bassins d'une écluse. » (Ph. Vasset, op. cit., p. 130-131).

11. Ph. Vasset, op. cit., p. 10.

12. Voir à ce propos l'article d'I. de Solà Morales Rubio, W.B. Campbell, L. Lévesque, «Urbanité interstitielle ", dans Inter : art actuel, 61, 1995, p. 27-28, où les chercheurs tentent de renverser la perspective selon laquelle on regarde habituellement ces espaces qui, de lieux de désolation, deviennent d'hypothétiques lieux d'espoir, voire des terrains d'expérimentation artistique (on pense à l'usage qu'en font les tagueurs).

13. «Lors de ma première visite, je n'ai pas réussi à déterminer quel genre d'activité avait abrité ce bâtiment, et je m'étais promis d'y revenir. C'est ce que je fis trois mois plus tard: les herbes avaient encore poussé, et je me perdis à plusieurs reprises. Mais, parvenu au bout du terrain, plus de maison : elle avait été entièrement rasée. Il n'y avait plus qu'un sol boueux mêlé de débris de tuile et labouré par les engins de démolition » (Ph. Vasset, op. cit., p. 32).

14. Ibid., p. 35.

15. "Chaque expédition se déployait comme un atterrissage. Avant de partir, il n’y avait, sur la carte, que des formes abstraites et des à-plats colorés, comme un paysage que la distance fige mais, au fur et à mesure que j'approchais, tout s'animait. C'était d'abord des mouvements vastes et lents, une circulation à peine perceptible, puis, progressivement, un grouillement continu » (Ibid., p. 33).

16. Voir l'interview accordée par l'auteur le 19/10/2007 et visible sur le site de l'INA http:// www.ina.fr/video/3470303001/philippe-vasset-un-livre-blanc-video.html.

17. Ph. Vasset, op. cit., p. 10.

18. Cf. Z. Bauman, Liquid Modernity, Cambridge/Oxford, Polity/Blackwell Publishers Ltd., 2000. Ce n'est pas un hasard si les expressions relatives à l'eau sont nombreuses dans le livre de Vasset.

19. Récemment encore, la périphérie évoquait une excroissance de la ville en cours d'aménagement autour de sa partie ancienne. Ce centre historique était ainsi considéré comme le modèle de référence vers lequel devait tendre l'urbanisation nouvelle. Or, il est à peu près admis aujourd'hui que la périphérie correspond à des morphologies, des paysages et des modes de vie différents, et que la périurbanisation diffuse oblige à s'interroger autant sur l'état que sur le sens de la ville. Voir sur le sujet Françoise Rouxel et Geneviève Brunet, Le périurbain ou les mutations de la ville périphérique, dossier documentaire, Paris, Centre de documentation de l'urbanisme, novembre 2002.

20. M. Augé, Non-lieux. Introduction à une anthropologie de la surmodernité, Paris, Seuil, 1992, p. 74. Il semble possible, en réalité, d'affiner le concept de "non-lieu » à partir même de Vasset. Car, comme le montre bien le travail de Jacques Lévy et Michel Lussault sur les "géotypes de l'urbanité » (central, périurbain, suburbain, méta-urbain, etc,), la banlieue est loin d'être un nonlieu, un espace défectif. Voir notamment leur Dictionnaire de la géographie et de l'espace des sociétés, réédité par l'éditeur parisien Belin en 2013.

21. Ph. Vasset, op. cit., p. 21.

22. Ibid., p. 18.

23. Ibid., p. 20.

24. Ibid., p. 34.

25. F. Martin-Achard, op. cit., p. 18.

26. Ph. Vasset, op. cit., p. 61.

27. Vasset écrit: "L'apparente passivité des zones blanches cache une sourde résistance au comblement: là, comme dans les marais, se brouillent les frontières entre le neuf et l'ancien, l'occupé et le vacant » (Ibid., p. 81).

28. F. Martin-Achard, op. cit., p. 24.

29. Ibid., p. 24.

30. Ibid., p. 116. 
31. Sur le sujet, voir R. Pasquier, op. cit., en particulier p. 76-77.

32. Indiquées par un vide sur la carte, ces zones vierges ne peuvent accéder à une existence que de façon paradoxale : elles sont sans cesse en porte-à-faux entre un temps passé et un temps à venir - celui de leur réhabilitation - non encore enregistrées par une représentation cartographique susceptible de les légitimer.

33. Cité dans « En quête du réel » par A. Djian, Le Monde des Livres, 27 février 2009.

34. R. Pasquier, op. cit., p. 73.

35. À ce sujet, voir la critique d'A. Maïsetti dans Carnets, Lectures, Littératures : Philippe Vasset|Un livre blanc, 11 mai 2008, consulté le 26/11/2015, URL: http://www.arnaudmaisetti.net/spip/ spip.php?article82.

36. Ibidem.

37. Ph. Vasset, op. cit., p. 104.

38. F. Martin-Achard, op. cit., p. 18.

39. Ph. Vasset, op. cit., p. 54.

40. Ibidem.

41. En écrivant le sien, Vasset se souvient de certains livres avec lesquels on peut errer à son propre gré, comme par exemple des romans de Claude Simon ou L'inquisitoire de Robert Pinget. Cf. R. Pasquier, La ville et ses fictions, cit., p. 80.

42. Voir les études de B. Westphal sur la représentation de l'espace dans les univers fictionnels, parmi lesquelles notamment La Géocritique. Réel, fiction, espace, Paris, Les Éditions de Minuit, 2007 et Le monde plausible. Espace, lieu, carte, Paris, Les Éditions de Minuit, 2011. Mais aussi, dans la même perspective multidisciplinaire : Piani sul mondo. Le mappe nell'immaginazione letteraria, édité par M. Guglielmi et G.. Iacoli, Macerata, Quodlibet, 2012, et Letteratura e geografia. Atlanti, modelli, letture, édité par F. Fiorentino et C. Solivetti, Macerata, Quodlibet, 2012.

43. F. Martin-Achard, op. cit., p. 18.

44. «Pour moi, c'était comme si le tamis de la carte avait tranché dans le vif de la ville, découvrant sa pulpe. Je n'étais pas là par hasard et pourtant, j'étais environné des scènes domestiques ne me concernant en rien : enfants jouant dans un parc, couples déchargeant leur voiture, jeunes roulant des joints sur un banc, etc. J'étais, voyeur sans objet, devenu transparent : libre de tout rôle, j'observais tout ce qui venait s'encadrer dans ma mire, sans rien décrire ni recenser [...]» (Ph. Vasset, op. cit., p. 64).

45. F. Martin-Achard, op. cit., p. 16.

46. Ibid., p. 27.

47. Compte rendu d'Un livre blanc par S. Alexandre le 15 mai 2009 dans Actu Philosophia, Coups de cœur, consulté le 26/11/2015, URL : http://www.actu-philosophia.com/spip.php?article100.

48. Ph. Vasset, op. cit., p. 136.

49. F. Martin-Achard, op. cit., p. 10.

50. Ph. Vasset, op. cit., p. 100.

51. Voir l'interview accordée par l'auteur le 19/10/2007 et visible sur le site de l'INA http:// www.ina.fr/video/3470303001/philippe-vasset-un-livre-blanc-video.html.

52. F. Martin-Achard, op. cit., p. 18.

53. Ibid., p. 26.

54. Dans son Walkscapes. Camminare come pratica estetica, Turin, Einaudi, 2006, F. Careri présente cette perception particulière de l'espace qui implique aussi bien le corps (marche à pied, sauts d'obstacles) que l'esprit (la mémoire des lieux traversés, la perception de leur utilisation sauvage). 


\section{RÉSUMÉS}

Explorateur débutant des espaces métropolitains ultramodernes, l'écrivain français contemporain Philippe Vasset s'est donné pour mission de parcourir pendant un an cinquante zones blanches de la carte de Paris $n^{\circ} 2314$ OT de l'IGN afin de découvrir ce qui se cache derrière ces espaces «vides » (non habités ou incultes, périphériques et marginaux aux yeux du pouvoir officiel), c'est-à-dire ce qui échappe à la représentation parce que retenu souvent comme non présentable. De fait, Vasset tente de combler, à l'aide d'une écriture qu'il définit lui-même de "spatiale », des lacunes dans la connaissance pour faire exister, littéralement, des non-lieux. Ainsi, particulièrement dans Un livre blanc (Fayard, 2007), cartographie et roman s'entrecroisent continuellement pour composer un "documentaire romancé » ou un "texte-performance » vraiment original : le compte rendu à la fois objectif et subjectif d'une enquête sociologique et littéraire dans les avant-postes de la mutation urbaine.

\section{INDEX}

Mots-clés : Vasset Philippe, littérature française de l'extrême-contemporain, écriture spatiale, enquête périurbaine, texte-performance, géographie parallèle 